\title{
Integration of Radio Frequency Identification with Programmable Logic Controller for Design of Industrial Control System
}

\author{
Richard Kapolyo, \\ Department of Automation and Electrical Engineering, Tianjin University of Technology and Education, \\ Tianjin 300222, People's Republic of China.
}

\begin{abstract}
This paper provides the method of integrating of Programmable Logical Controller (PLC) with Radio Frequency Identification (RFID) technology for the design of industrial control system. Today's competitive worldwide economy, manufacturing operations must react quickly and flexibly to meet customer demands while maintaining or increasing competitiveness with regard to their own production costs. One potential solution involves integration Radio frequency identification systems (RFID) with Programmable Logic Controller in the manufacturing process infrastructure in order to facilitate an expanding degree of customization while keeping production costs stable. The method of RFID integration with PLC system requires a comprehensive knowledge and understanding of different systems. The method is achieved through the understanding of both software, and hardware configuration. The aim of integration of PLC and RFID is basically to enhance the speed of data flow; this is because RFID offers many features which make it viable for efficient integration in variety of Industrial, consumer and commercial concerns. These data are queried from the production control system at the start of a production line and programmed on the transponder. PLC controllers at the individual manufacturing stations read these data directly from RFID readers and use them to control the production step. It's a technology that has opened up a wide range of new opportunity in direct and integrated form for different technical fields and areas.
\end{abstract}

Keywords: PLC with RFID Integration, industrial control system design

\section{Introduction}

There exist a number of industrial control systems where Programmable Logic Controller (PLC) has been applied. For example, main assembly lines in the automotive industry such as body shop, conveyor systems for the assembly lines, it's used for automation of typically industrial electromechanically factory assemblies, this is due to its good versatility and high reliability [1].

The integration of Siemens Programmable Logic Controller (PLC) with Radio Frequency Identification (RFID) is important in that it ensures constant flow of information, smooth and efficient processes, quick transfer of data, and more data can be delivered to the monitoring personnel without time delay. However, RFID integration with PLC system requires a comprehensive knowledge and understanding of different systems. Functioning and reliable RFID systems, requires knowledge of business, physics and RFID system design. To be able to integrate RFID functions within a system and with other technologies like PLC such as vision, scan, control and information technologies requires the experience of hardware and technology integration. In applications which require input of masses such as contactless ticketing, to mention a few, social and society values and norms also gains important consideration [2].

The contactless data transmission guarantees a high suitability for industrial conditions and large scale implementation. The integration of RFID with PLC for design of industrial control system is considered one of the most promising technologies in twenty-first Century, and it will be widely used in many fields. It will change people's ways of living and build a new mode of future retail and logistics. The integration of PLC with RFID in the networking industry will help in development of information industry, and this technology will be used in automatic control and management of database industry fields. In the whole supply chain logistics, sales of industrial warehousing plays a crucial role, correct stock and inventory control and delivery will be conducive to the control of the management cost, guarantee of quality of services as to enhance the enterprise competitiveness. The paper presents one way of integrating Siemens S7-300 PLC with RFID system of reader, and tag communicating at $13.56 \mathrm{MHz}$ [3].

\section{Basics of RFID System}

Radio Frequency Identification (RFID) makes it possible to automatically identify and localize objects and living beings, and thus considerably facilitates recording and storing data. In general a RFID system has a structure as depicted in figure 1. RFID readers scan tags, and then forward the information to the backend. The backend in general consists of a database and a well defined application interface. When the backend receives new information, it adds it to the database and if needed performs some computation on related fields.

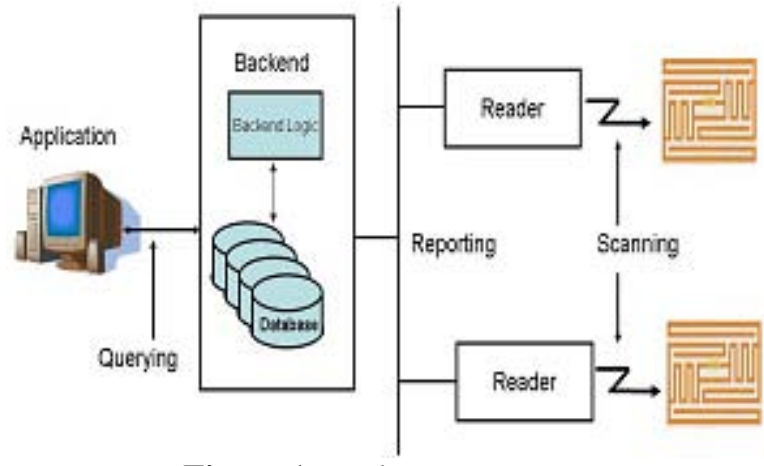

Figure 1: Basic RFID system 


\section{Basic Principle of RFID}

The RFID system consists of the following: (1) a transponder that is located in the object or in the living being and identifies it, and (2) a reading device for reading out the transponder ID.The reading device includes software that controls the actual read process, and an RFID middleware with interfaces to other Electronic Data Processing (EDP) systems and data bases. As a rule, a read device generates an electro-magnetic high frequency field with a short range, preferably with induction coils. It is not only used to transmit data, but also to supply the transponder with power, here the frequency of $13.56 \mathrm{MHz}$ is used according to the International Standard Organizational). The reading device (reader) generates a high frequency electromagnetic alternating field that illuminates the aerial of the RFID transponder (RFID tag). As soon as the aerial coil enters the electro-magnetic field, an induction current is generated in it. This current is rectified, and with it, a capacitor is loaded as short time storage which, for the read process, provides for the power supply of the chip. For active tags, an installed battery takes care of the supply. The micro-chip thus activated in the RFID tag decodes the commands sent by the reader. This reader encodes and modulates the reply into the irradiated electro-magnetic field through field weakening in the contact-free short circuit, with this; the tag transmits its own unchangeable serial number, additional numbers of the marked object. The transponder itself does not transmit a field; it only changes the reader's electro-magnetic transmission field.

\section{(a) Components Required For RFID Integration With Other Systems}

RFID composed of reader and tag for the user, but mainly it is integrated with other systems to perform the desired task. This makes much small hardware or software components, which are considered less thoughtful to gain more importance especially in large scale industrial and commercial applications. Figure 3 below show some components which are needed to be considered in RFID system integration.

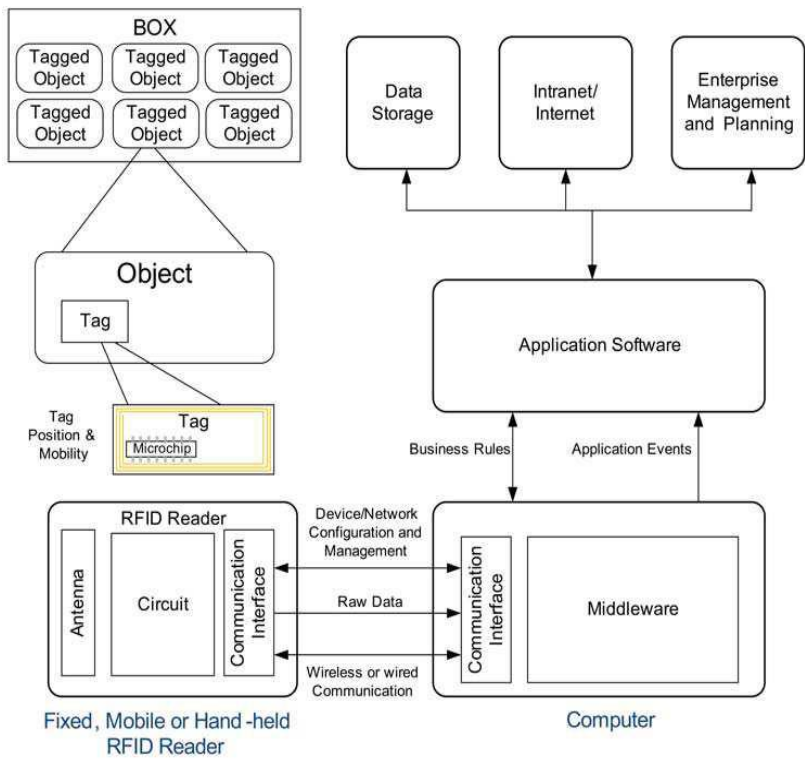

Figure 3: Main components to consider when RFID Integration with other systems

\section{PLC Integration With RFID}

\section{(a) Simplified System Interconnection}

Programmable logic controller (PLC) is a specialized industrial computer used for automation of real-world processes. Most of the leading processes, oil and gas, food, beverage, and similar manufacturing companies have sophisticated automation systems in place that control the high-speed packaging lines in their manufacturing operations, and high-speed conveyors in their distribution facilities .These systems are typically controlled by Programmable Logic Controllers (PLCs), which are dedicated automation systems programmed and maintained by electricians or technicians [5].

As Radio Frequency Identification (RFID) becomes more ubiquitous, is integrated with the mainstream production process with PLC architecture enabling engineering managers to maintain and upgrade the incremental RFID infrastructure using their existing personnel skill set. RFID is widely adopted in automotive industry. An RFID tag is attached to each vehicle or skid and programmed in production line; this data can be read out and processed directly by means of a PLC. At each manufacturing station, this data is remotely read out by the PLC and then processed to control the production step with PLC updating the data or status information on the RFID tag at end of each station. Figure 4 below shows the basic block diagram for PLC with RFID integration system [6].

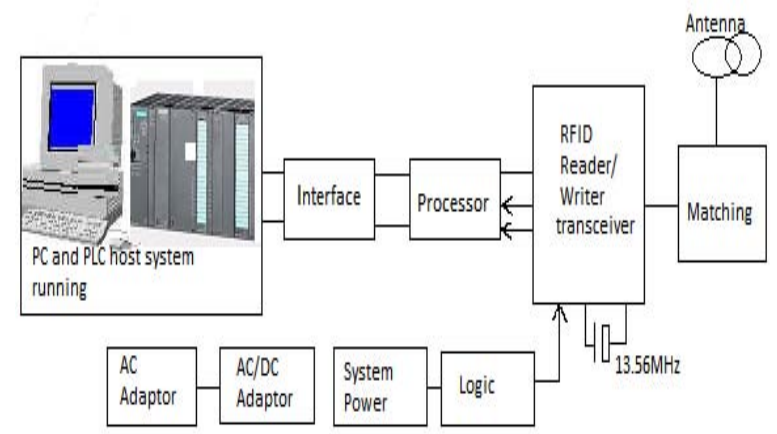

Figure 4: Block diagram of the system

The operating frequency will help in determining how the tags will interact with each other. An RFID reader for communicating with an RFID tag, and with a remote RF transceiver. Single transceiver is employed for communicating with RFID tags and with a remote RF transceiver. A single antenna is coupled to the transceiver. In a first mode, the transceiver communicates with the RFID tags through the antenna, on a first frequency. In a second mode, the transceiver communicates with the remote $\mathrm{RF}$ transceiver through the same antenna, on the first frequency or a second Frequency.PLC is computer-based solid-state devices that control industrial equipment and processes Siemens S7-300 PLC is used to provide regulatory control of discrete processes. It is often the primary components in smaller control system configurations. The proportional, integral, and differential gains of the PLC continuous control feature may be tuned to provide the desired tolerance as well as the rate of self-correction during process upsets. RFID system power is determined by the type of tags used [7]. 


\section{International Journal of Science and Research (IJSR) \\ ISSN (Online): 2319-7064 \\ Index Copernicus Value (2013): 6.14 | Impact Factor (2015): 6.391}

(b) Implementation of Serial Communication Between PLC and RFID

Programmable Logic controller (PLC) serial communication through the serial I/O communication protocol Radio Frequency identification reader, computer serial communication, and the windows multi-threading technology and RFID reader is easier to control in the field of automation in the process of communication, data acquisition. The RFID tag stores non volatile memory data, On the other hand, do not need to see a direct RFID this is because RFID is using radio communication, therefore, need only label range at the reader. To pay attention to the PLC write identification data only need to perform write the serial command on it, while the PLC read identification data process involves two steps:
First, write the serial PLC execution command, write read identifies the command to the RFID reader; PLC executes the read serial command to capture the data returned by the RFID reader. This is due to the RFID reader identifies the received read command, read command returns a response message to the serial buffer, which contains the read identification data. Reader indicator changes secondary PLC program debugging. As PLC, the CPU 318-2 DP and as RFID, a SIMATIC RFID system is used [8].

The table 1.below shows the common method of communication standards used today between the RFID reader and the computer. In this paper the Ethernet communication standard is used.

Table 1: Different communications standards used today between reader and computer

\begin{tabular}{|c|l|l|l|}
\hline Standard & Range (m) & Data Rate & Remarks \\
\hline RS-232 & 15 & $20 \mathrm{Kbps}$ & $\begin{array}{c}5 \text { or } 9 \text { serial wire } \\
\text { communication }\end{array}$ \\
\hline RS-485 & 1200 & $\begin{array}{c}3 \mathrm{Mbps} \text { upto } 100 \mathrm{Kbps} \text { at } \\
1200 \mathrm{~m}\end{array}$ & $\begin{array}{c}\text { 2wire half, duplex multiple serial } \\
\text { communication }\end{array}$ \\
\hline USB & 5 & 12 or $80 \mathrm{Mbps}$ & $\begin{array}{l}\text { most wide used computer } \\
\text { connection method }\end{array}$ \\
\hline Ethernet & 100 to 200 & $10 / 100 / 1000 \mathrm{Mbps}$ & widely used long range \\
\hline Zigbee & $10 \sim 75$ & $20 / 40 / 250 \mathrm{Kbps}$ & for wireess applications \\
\hline WiFi & $32 \sim 95$ & 11 and $54 \mathrm{Mbps}$ & $\begin{array}{l}\text { wirelss technolgy supported by } \\
\text { computers, mobile phones tec }\end{array}$ \\
\hline Profibus & & $31 \mathrm{Kbps}$ and $12 \mathrm{Mbps}$ & popular fieldbus in process industry \\
\hline
\end{tabular}

(c) The Software and Hardware Required For Integration of PLC and RFID

The integration of RFID and PLC systems require software that manages devices, networks, data and processes to enable continual information flow, alerts, decision support and realtime response to an existing host. Application software is usually designed with function libraries, function blocks or drivers for the quick and easy integration into the respective system. Tools, libraries, API's (application programming interfaces) speeds up the integration of RFID equipment in to existing enterprise system regardless of equipment type, manufacturer, enterprise system application or operating environment. This shields system integrators from the details of low-level protocols, standards, and proprietary hardware interfaces, providing instead a single API for a wide range of hardware. The S7-300 Automation system hardware of central processing unit (CPU) 318-2DP is used, and it is shipped with 2 integrated interfaces .Computer having operating system Windows XP Professional with SP2 and Business/Server 2003 SP2 with $600 \mathrm{MHz}$ (only XP)/1 GHz and 1 or 2 GB RAM, free disk storage. Programmable logic controller software SIMATIC S7-300 with central processing unit 318-2DP and at least one digital Input and output module Sample configuration, Power supply from PS 307 2A at $24 \mathrm{~V}$,central processing unit 318-2DP,digital inputs of $16 \times 24 V$ DC digital outputs of $16 \times 24 \mathrm{~V}$ DC/0.5 A4,and the SIMATIC RF180C IM (Interface Module),RF310R [9].

\section{(d) Connection of Ethernet Cable Between Computer,} PLC and Reader

The SIMATIC RF180C reader with Ethernet interface should be connected to a SIMATIC S7-300 controller. For interface with PLC, RFID reader has to communicate with standard module which can be plugged in to the backplane of PLC rack. Usually module communicates data between the RFID Tags and the host PLC through a simple ladder logic program in the PLC. The program in module offers normal operations such as Reading and Writing to a Tag and returning status of operations to the PLC. Serial port is usually available with such modules to download and debug the software. These industrial modules are designed to read/write multiple RFID readers, some have ports for antenna and reader resides in module. To have compatibility with PLC system, the module has 24VDC supply. Figure 5 below shows the layout diagram of the Ethernet connection between PC, CPU318-2DP and RF180C [11].

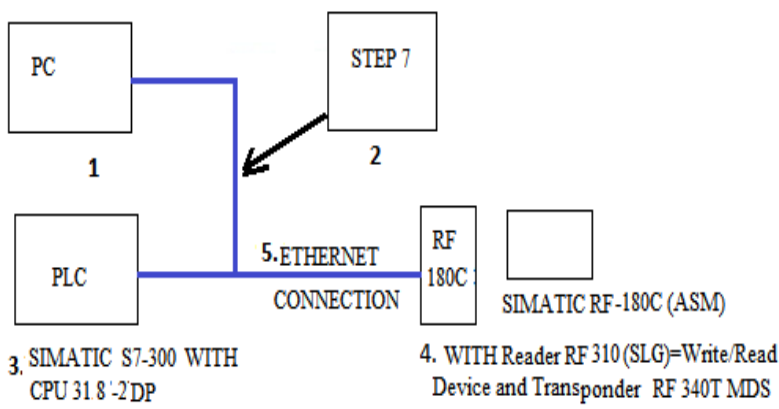

Figure 5: Ethernet connection between PC, CPU318-2DP and RF180C

The Central processing unit 318-2DP is used as the controller for the data exchange of a S7-300 and RFID system on the PROFINET. To run this CPU, a micro-memory card is required. The addresses of the input and output modules can 


\section{International Journal of Science and Research (IJSR) \\ ISSN (Online): 2319-7064 \\ Index Copernicus Value (2013): 6.14 | Impact Factor (2015): 6.391}

be parameterized at this CPU.The first interface is a combined MPI/PROFIBUS DP interface that can be used at the PROFIBUS DP as master or slave to connect distributed input or output field devices with very fast reaction time. In addition, it is possible to program the Central processing unit here by means of an MPI. The second interface is an integrated PROFINET interface. This allows for using the Central processing unit as PROFINET input or output controller to operate distributed input or output on PROFINET. The Central processing unit can be programmed by means of this interface. Moreover, it is possible to use fail-safe input or output devices on both interfaces [10].

\section{Data Flow Between RFID and PLC}

Apart from insensitivity to many of the environmental influences that hamper visual codes, a second advantage of RFID is the possibility of rewriting the data carriers. Once printed, a 2D code cannot be changed. Together with the high memory capacity of RFID transponders (up to 32 Kbytes), remote automation architectures can be realized, which clearly reduces the effort for local data maintenance. These data are queried from the production control system at the start of a production line and programmed on the transponder. PLC controllers at the individual manufacturing stations read these data directly from RFID readers and use them to control the production step [12].

\section{Conclusion}

The paper has presented the method of integrating Programmable Logical Controller Siemens s7-300 and the Radio Frequency Identification which is based on the reader and tag communicating at $13.56 \mathrm{MHz}$. The integration is done for the design of industrial control system.PLC S7-300 offers the controllers at the individual manufacturing stations read the data directly from RFID readers and use them to control the production step. After the production step is completed, the PLC can store the status and quality data on the RFID transponder before it is transported to the next station with the work piece. Such a concept provides considerable advantages: the individual stations can perform their manufacturing step autonomously. Central planning and control is only required at the start of the manufacturing line, when the transponders are initialized. The complexity in the automation systems and in the engineering of such factors, therefore, decreases. Reducing complexity is equivalent to decreasing the susceptibility to failure for the entire plant. The integration of Siemens PLC with RFID is important in that it ensures constant flow of information, smooth and efficient processes, quick transfer of data, and this technology in the networking industry will help in the development of information industry. Finally, the integration of Radio Frequency Identification and the Programmable Logic Controller will also be used in automatic control and management of database industry fields.

\section{Future Scope}

The use of PLC and RFID technologies for the design of industrial control systems has increased in the modern world. The aim of integration of PLC and RFID is basically to enhance the speed of data flow; this is because RFID offers many features which make it viable for efficient integration in variety of Industrial, consumer and commercial concerns. These data are queried from the production control system at the start of a production line and programmed on the transponder. In fact, the technology has moved from obscurity into mainstream applications that help speed up the handling of manufactured goods and materials .Because the use of RFID enables identification from a distance, and unlike the earlier bar-code technology, it does so without requiring a line of sight. There are possibilities that the use of this technology will continue expanding since there is increase in the number of companies manufacturing the products that are required to use in system integration. However, the use of this technology has taken a long time to develop this is due to some challenges that are needed to be addressed .For example, the use of RFID isn't cheaper as traditional labeling technologies. It has reached the critical price point that could not enable its large-scale adoption for managing consumer retail goods. In additional, Siemens PLC both hardware and software are costly. More ever, the use of RFID technology requires technical, process and security issues to be solved -ahead of time, and RFID software maybe attacked by the slammer virus.

Finally, since there is an increase in the number of manufacturing companies that supplies both PLCs and RFID products which are used in the system integration, there are higher possibilities to the price reduction of the products, and increase in the use of this technology, and specialists are already working in several of these issues to overcome the problems.

\section{References}

[1] W. Bolton ,Programmable Logic Controller: fifth edition , 2000.

[2] G.M. Regains the Top Spot in Global Auto making," Business Day, New York Times, January 19, 2012.

[3] [3] J. Landt, Shrouds of time-The history of RFID," AIM

[4] G. R Paul,. (2007). Engineering Management-Focused Radio Frequency Identification (RFID) Model Solutions, IEEE Engineering Management Review, vol. 35, no. 2, Second Quarter, 2007, ISSN: 0360-8581.

[5] Cox. Jr.; George, D. T. (2007). System and method for RFID System Integration, Unites States Patent, US 7267275 B2, Date of patent Sep. 11, 2007.

[6] Qiu, R. G. (2007). RFID-Enabled Automation in Support of Factory Integration, Robotics and computerintegrated manufacturing, 16th International Conference on Flexible Automation and Intelligent Manufacturing, December 2007.

[7] Hsu, Y. C.;Chen, A. P.;Wang, C. H. (2008). A RFIDenabled Traceability System for the Supply Chain of Live Fish, IEEE International Conference on Automation and Logistics,

[8] H. Stockman, -Cmmunication by means of reflected power," Proc.IRE, Oct. 1948

[9] Karthikeyan, S.; Nesterenko, M. (2005). RFID Security without Extensive Cryptography, TheThird CCS ACM Workshop on Security of Ad Hoc and Sensor Networks (SASN'05), Washington, DC, USA, November, 2005 


\section{International Journal of Science and Research (IJSR) \\ ISSN (Online): 2319-7064}

Index Copernicus Value (2013): 6.14 | Impact Factor (2015): 6.391

[10] Jabbar, H.; Jeong, T.; Hwang, J.; Park. G. (2008). Viewers Identification and Authenticaion inIPTV using RFID Technique, IEEE Transactions on Consumer Electronics, vol. 54, Feb. 2008.

[11]Dong Geun Yoon, Dong Hyeon Lee, Chang HoSeo, SeongGon Choi, - RHD NetworkingMechanism Using Address Management Agent," 4th Networked Computing and Advanced Information Management(NCM2008), vol. 1, Sept. 2008

[12] Yang, L., et al., Đesign and Development of Novel Inductively Coupled RFID Antennas,"Atlanta, GA: School of Electrical and Computer Engineering, Georgia Institute of Technology, 2006. 